\title{
Anomalias congênitas na perspectiva da vigilância em saúde: compilação de uma lista com base na CID-10
}

doi: 10.1590/S1679-49742020000500015

\author{
Congenital anomalies from the health surveillance perspective: compilation of a list based on \\ ICD-10
}

\section{Anomalías congénitas desde la perspectiva de la vigilancia de la salud: compilación de una lista basada en la CIE-10}

\author{
João Matheus Bremm - Đ orcid.org/0000-0002-2150-9426 \\ Augusto César Cardoso-dos-Santos ${ }^{1}$ - @ orcid.org/0000-0002-1499-9105 \\ Vivyanne Santiago Magalhães ${ }^{2}$ - (1) orcid.org/0000-0002-9540-4619 \\ Ana Cláudia Medeiros-de-Souza ${ }^{2}$ - orcid.org/0000-0001-9317-6424 \\ Ronaldo Fernandes Santos Alves ${ }^{2}$ - orcid.org/0000-0002-8358-0519 \\ Valdelaine Etelvina Miranda de Araujo² - (1) orcid.org/0000-0003-1263-1646 \\ Eduardo Marques Macario² - (1) orcid.org/0000-0002-6383-0365 \\ Wanderson Kleber de Oliveira ${ }^{2}$ - (1) orcid.org/0000-0002-9662-1930 \\ Lavínia Schüler-Faccini' ${ }^{1}$ - (D orcid.org/0000-0002-2428-0460 \\ Maria Teresa Vieira Sanseverino ${ }^{1}$ - @ orcid.org/0000-0002-7404-2911 \\ Giovanny Vinícius Araújo de França ${ }^{2}$ - Đ orcid.org/0000-0002-7530-2017 \\ 'Universidade Federal do Rio Grande Sul, Departamento de Genética, Porto Alegre, RS, Brasil \\ ${ }^{2}$ Ministério da Saúde, Secretaria de Vigilância em Saúde, Brasília, DF, Brasil
}

\section{Resumo}

Objetivo: Propor uma lista de anomalias congênitas com códigos correspondentes na Classificação Estatística Internacional de Doenças e Problemas Relacionados à Saúde - 10ª Revisão (CID-10), visando a aplicação no âmbito da vigilância em saúde. Métodos: Em dezembro de 2019, realizou-se busca nas seguintes fontes de dados: CID-10; CID-11; anomalias monitoradas por três modelos de vigilância; base de informações sobre doenças raras (Orphanet). Realizou-se extração das anomalias a partir dessas fontes, processamento para correspondência com base na CID-10 e compilação mediante revisão manual. Resultados: Foram identificados 898 códigos, dos quais 619 (68,9\%) constavam no capítulo XVII da CID-10. Dos 279 códigos de outros capítulos, 19 foram exclusivos da busca na CID-11, 72 dos modelos de vigilância, 79 da Orphanet e 36 da busca de termos na CID-10. Conclusão: 0s códigos que constam do capítulo XVII da CID-10 não captam a totalidade das anomalias congênitas, indicando a necessidade de adoção de uma lista ampliada.

Palavras-chave: Anormalidades Congênitas; Doenças Raras; Classificação Internacional de Doenças; Monitoramento Epidemiológico.

Endereço de correspondência:

João Matheus Bremm - Ministério da Saúde, Secretaria de Vigilância em Saúde, Unidade Técnica de Vigilância das Anomalias Congênitas, SRTVN 701, W5 Norte, Ed. PO 700, 6º andar, Brasília, DF, Brasil. CEP: 70723-040

E-mail: joao.bremm@saude.gove.br 


\section{Introdução}

A Organização Mundial da Saúde (OMS) define anomalias congênitas (defeitos congênitos) como alterações estruturais ou funcionais no embrião ou feto, derivadas de fatores anteriores ao nascimento, possíveis de se identificar no acompanhamento pré-natal, no nascimento ou mais tarde, ao longo da vida. ${ }^{1}$ No mundo, estima-se cerca de $3 \%$ dos nascidos vivos com alguma anomalia, a cada ano, ${ }^{2,3}$ sendo que pelo menos 3,3 milhões de crianças menores de 5 anos morrem anualmente, por causa de anomalias congênitas graves. Entre os afetados que sobrevivem, muitos apresentam incapacidades durante a vida. ${ }^{4}$

Um sistema de codificação e classificação abrangente e robusto é essencial para a vigilância de anomalias congênitas, porém não existe uma lista acordada que reúna todos esses agravos. ${ }^{5} \mathrm{~A}$ Classificação Estatística Internacional de Doenças e Problemas Relacionados à Saúde (CID) constitui a codificação adotada internacionalmente, para propósitos epidemiológicos. Em sua décima revisão (CID-10), seu capítulo XVII (Malformações congênitas, deformidades e anomalias cromossômicas) faz referência exclusivamente a anomalias congênitas. ${ }^{6}$ Apesar de esse capítulo ser amplamente utilizado como referência para vigilância, existem outros agravos que se enquadram no conceito de anomalias congênitas mas se encontram em outros capítulos da mesma CID-10. A despeito da atualização realizada em sua décima primeira edição, CID-11, instituições internacionais, como a International Clearinghouse for Birth Defects Surveillance and Research (ICBDSR) e a European Network of PopulationBased Registries for the Epidemiological Surveillance of Congenital Anomalies (EUROCAT), apontam que a última versão não será suficiente para englobar todas as anomalias congênitas em capítulo único. ${ }^{5}$

\section{Um sistema de codificação e classificação abrangente e robusto éessencial para a vigilância de anomalias congênitas, porém não existe uma lista acordada que reúna todos esses agravos.}

Alguns programas internacionais de vigilância de anomalias congênitas incluem condições ausentes do capítulo XVII da CID-10, segundo seus objetivos e prioridades. No Brasil, os códigos do capítulo XVII da CID-10 são utilizados para registro dos casos de anomalias congênitas em sistemas de informações, a exemplo do Sistema de Informações sobre Nascidos Vivos (Sinasc). A proposição de uma lista utilizandose de múltiplas fontes de informação visa permitir 0 estabelecimento de um conjunto ampliado de anomalias congênitas, de maneira a elencar aquelas prioritárias para monitoramento. Desta forma, será possível expandir o rol de códigos habilitados para inserção no sistema e, por conseguinte, aprimorar a vigilância de anomalias identificadas no nascimento.

Neste trabalho, objetivou-se propor uma lista expandida de anomalias congênitas para além do capítulo XVII da CID-10, visando a sua disponibilização para aplicação no âmbito da vigilância em saúde.

\section{Construção da lista}

Foram utilizadas as seguintes fontes de informação: CID-10, publicada em 2007 e vigente no Brasil; CID11, lançada pela OMS em 2019, com o propósito de ser implementada a partir de 2022; anomalias congênitas monitoradas por modelos de vigilância selecionados, identificados a partir de uma revisão narrativa da literatura; ${ }^{7}$ e um repositório/base de informações sobre doenças raras, o 0 rphanet. ${ }^{8}$ Realizou-se a extração dos códigos das diferentes fontes em dezembro de 2019.

Foram incluídas condições que atendessem ao conceito de anomalias congênitas adotado pela OMS. ${ }^{1}$ Embora não sejam anomalias congênitas por definição, as infecções congênitas foram consideradas, haja vista algumas apresentarem desfechos importantes no contexto da vigilância. ${ }^{9}$ Foram excluídas condições sobre as quais não havia codificação correspondente na CID-10, especialmente para manter correspondência com o registro dos casos nos sistemas de informações do Ministério da Saúde.

0s códigos das anomalias congênitas do capítulo XVII foram captados da CID-10. Também foi realizada busca, pelos termos 'congênit', 'malforma', 'displas', 'disrup' e 'genétic', sendo considerados todos os códigos correspondentes.

As anomalias congênitas da CID-11 foram extraídas do capítulo 20, que compila os defeitos do desenvolvimento, bem como de todos os códigos relacionados aos erros inatos do metabolismo anomalias congênitas por definição. 
Utilizaram-se, ainda, as listas de anomalias de três modelos de vigilância: EUROCAT, ${ }^{10,11}$ ECLAMC (Latin American Collaborative Study of Congenital Malformations) ${ }^{12,13}$ e CREC (Costa Rican Birth Defects Register). ${ }^{14}$ As redes ECLAMC e EUROCAT foram selecionadas por serem referência para a AméricaLatina e a Europa, respectivamente. 0 CREC foi escolhido por incluir um grande número de anomalias funcionais e vigiar a maior quantidade de anomalias congênitas fora do capítulo XVII da CID-10, comparado aos demais programas analisados. ${ }^{13} 0$ ECLAMC não possui lista específica de anomalias para vigilância, mas fornece um atlas de consulta, dotado de uma lista definida de condições. ${ }^{13}$

A partir da Orphanet, ${ }^{8}$ foram aplicadas duas estratégias para extração das anomalias congênitas. $\mathrm{Na}$ primeira, entre as 35 classificações de doenças raras, seis atendiam ao conceito de anomalias congênitas: (i) Defeito de desenvolvimento raro durante a embriogênese; (ii) Malformações cardíacas raras; (iii) Erros inatos e raros do metabolismo; (iv) Doenças genéticas raras; (v) Desordens teratogênicas raras; e (vi) Anomalias cromossômicas classificadas por cromossomos. A segunda estratégia de busca procurou captar anomalias congênitas além dessas classificações, a partir do modelo de classificação hierárquica do tipo de desordens (http:/www.orpha.net/orphacom/cahiers/docs/GB/ Orphanet_linearisation_rules.pdf), considerando-se como anomalias congênitas as síndromes malformativas e anomalias morfológicas.

Os códigos das anomalias congênitas da CID-11 e da Orphanet foram mapeados, para correspondência com a CID-10, segundo orientação das próprias fontes. Da Orphanet, foram captados os códigos que mantinham correspondência exata com a CID-10. Posteriormente, as anomalias congênitas captadas foram compiladas em uma lista única, retirando-se as duplicatas.

Após processamento, a lista foi revisada manualmente (Bremm JM e Cardoso-dos-Santos AC), para exclusão das condições que não se enquadravam no conceito de anomalias congênitas. A lista obtida após essa revisão foi validada por duas geneticistas, especialistas na área (Sanseverino MTV e Schüler-Faccini L). Por fim, para classificação das anomalias na lista final, adotou-se 0 modelo proposto na CID-11, uma classificação mais atual e abrangente que a CID-10.
A Figura 1 apresenta as fontes consultadas e 0 processo para obtenção dos dados. Da CID-10, além dos 619 códigos do capítulo XVII, foram captados 509 mediante a busca por termos; feita a compilação de ambos os conjuntos e retiradas as duplicatas, chegouse a 701 códigos. Na CID-11, o capítulo 20 (Anomalias do Desenvolvimento) conta com 1.311 códigos de anomalias congênitas, além dos 193 relacionados aos erros inatos do metabolismo, que, após correspondência com a CID-10, resultaram em 683 códigos únicos. Em relação aos modelos de vigilância, foram incluídos 638 códigos a partir do EUROCAT, 650 do ECLAMC e 731 do CREC, logo compilados e deduplicados, resultando em 767 códigos únicos.

Entre as classificações da Orphanet, foram captados códigos referentes aos defeitos de desenvolvimento raros durante a embriogênese (3.507 códigos), malformações cardíacas raras (242), doenças genéticas raras (6.520), erros inatos e raros do metabolismo (1.058), desordens teratogênicas raras (39) e anomalias cromossômicas classificadas por cromossomos (511 códigos). Quanto ao tipo de desordens, as síndromes malformativas apresentaram 1.940 códigos, enquanto as anomalias morfológicas, 426. Uma vez compilados e de duplicados os códigos captados da Orphanet, foram excluídos 6.498. Dos 7.295 códigos traduzidos dessa base para a CID10 , apenas 289 (3,96\% do total) apresentaram correspondência exata.

Em seguida, a lista de anomalias codificadas na CID-10, oriunda de diferentes fontes, foi compilada e deduplicada e 1.500 códigos foram excluídos. 0s 945 códigos restantes passaram por revisão manual, sendo 47 definitivamente excluídos por não se enquadrarem no conceito de anomalias congênitas. Esse processo resultou em uma lista com 898 códigos de anomalias, logo classificados em 21 grupos distintos, de acordo com a proposta da CID-11 (Figura 2).

A lista final, conclusiva deste trabalho, reuniu os 619 códigos do capítulo XVII da CID-10, além de 279 códigos de outros capítulos, dos quais 19 foram exclusivos da busca na CID-11, 72 dos modelos de vigilância, 79 da Orphanet e 36 da busca de termos na CID-10. Particularmente, 87 anomalias congênitas foram compartilhadas entre, pelo menos, duas dessas diferentes fontes (Figura 3). 


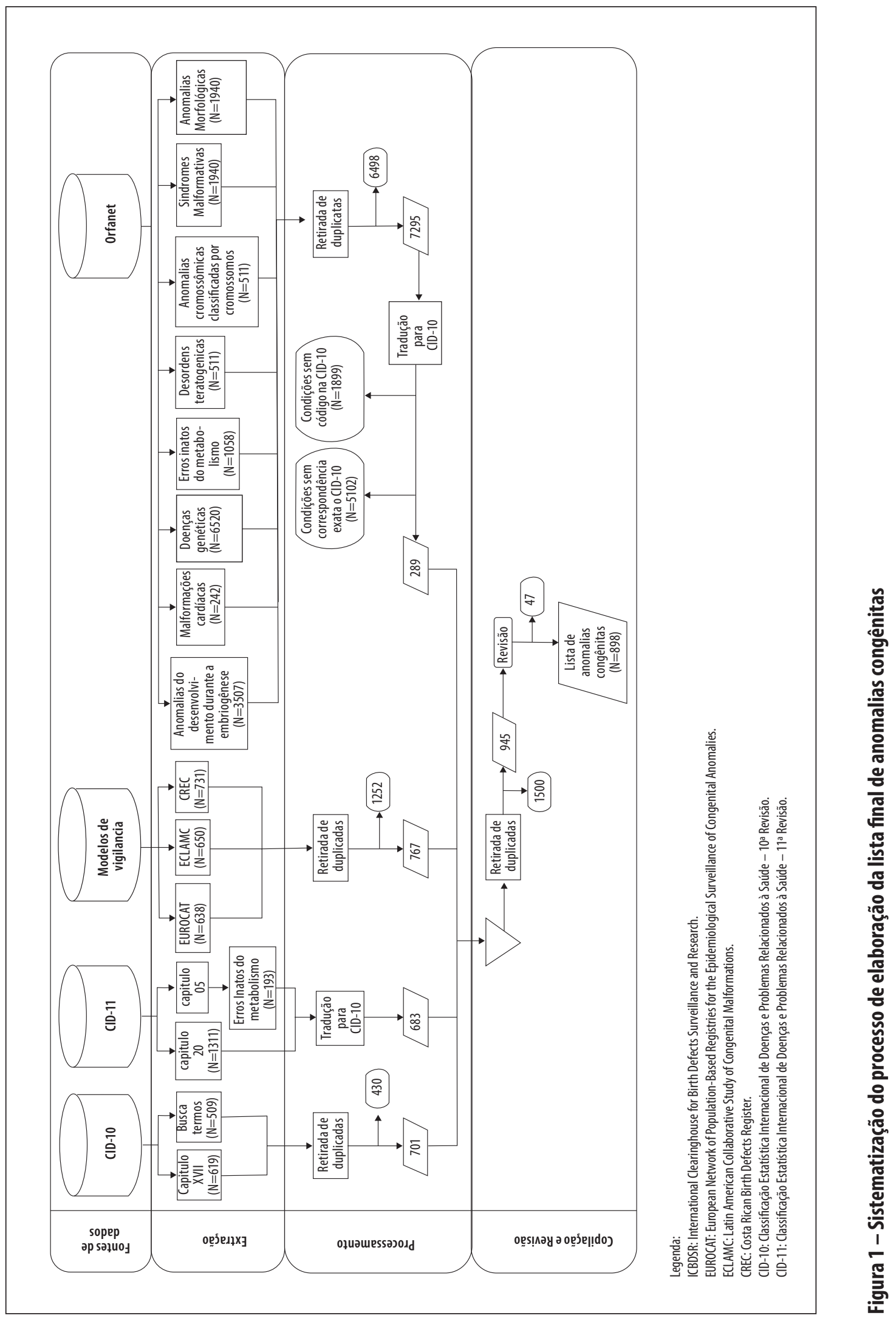




\begin{tabular}{|c|c|}
\hline Classificação das anomalias & Códigos das anomalias \\
\hline Certas doenças infecciosas ou parasitárias & A500, A501, A502, A503, A504, A505, A506, A507, A509, B004 \\
\hline Neoplasias & D179, D180, D181, D215, D220, D221, D222, D223, D224, D225, D226, D227, D229, L722, Q822 \\
\hline $\begin{array}{l}\text { Doenças do sangue ou órgãos formadores } \\
\text { de sangue }\end{array}$ & $\begin{array}{l}\text { D551, D552, D553, D560, D561, D562, D564, D572, D580, D581, D588, D595, D640, D644, D66, D67, } \\
\text { D680, D681, D740, D820 }\end{array}$ \\
\hline Doenças do sistema imunológico & $\begin{array}{l}\text { D720, D804, D806, D807, D808, D810, D811, D812, D813, D814, D815, D816, D817, D822, D823, D824, } \\
\text { D841 }\end{array}$ \\
\hline $\begin{array}{l}\text { Doenças endócrinas, nutricionais ou } \\
\text { metabólicas }\end{array}$ & 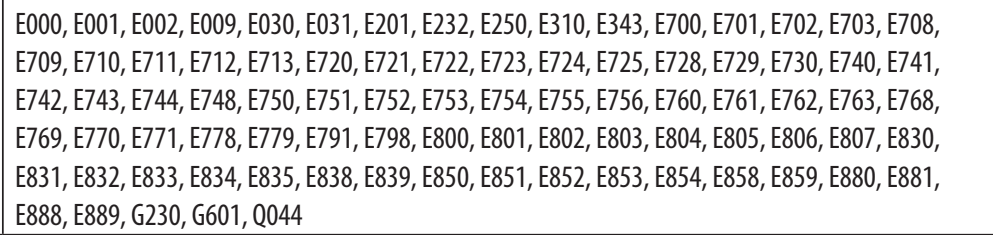 \\
\hline $\begin{array}{l}\text { Transtornos mentais, comportamentais ou } \\
\text { do desenvolvimento neurológico }\end{array}$ & F799, F841, F843 \\
\hline Doenças do sistema nervoso & $\begin{array}{l}\text { F803, G10, G110, G113, G114, G120, G231, G241, G244, G318, G404, G408, G512, G602, G633, G702, } \\
\text { G710, G711, G712, G800, G901, Q018, Q282 }\end{array}$ \\
\hline Doenças do sistema visual & H046, H052, H320, H494, Q150 \\
\hline Doenças do ouvido ou processo mastoide & H905 \\
\hline Doenças do sistema circulatório & I270, I424, I425, I429, I471, I498, I517, I773, Q820 \\
\hline Doenças do sistema respiratório & E840, E841, E848, E849 \\
\hline Doenças do aparelho digestivo & $\begin{array}{l}\text { K006, K008, K035, K068, K070, K071, K079, K088, K108, K116, K400, K401, K402, K403, K404, K409, } \\
\text { K420, K421, K429, K469, K552, K768, K831 }\end{array}$ \\
\hline Doenças da pele & $\begin{array}{l}\text { E882, L050, L059, L440, L631, L813, L818, Q800, Q801, Q802, Q803, Q804, Q810, Q811, Q812, Q818, } \\
\text { Q819, Q841, Q842, Q843, Q844 }\end{array}$ \\
\hline $\begin{array}{l}\text { Doenças do sistema músculo-esquelético } \\
\text { ou tecido conjuntivo }\end{array}$ & M111, M124, M162, M163, M231, M611, M850, M911, M918, M941, Q781 \\
\hline Doenças do aparelho geniturinário & N046, N251, N47, N948, Q611, 0612, 0613, 0615, 0618, 0619 \\
\hline $\begin{array}{l}\text { Certas condições originárias do período } \\
\text { perinatal }\end{array}$ & $\begin{array}{l}\text { P026, P113, P230, P231, P232, P233, P234, P235, P236, P238, P239, P271, P350, P351, P352, P353, } \\
\text { P358, P359, P370, P371, P373, P374, P378, P379, P560, P613, P614, P702, P745, P832, P835, P941, } \\
\text { P942, P960 }\end{array}$ \\
\hline $\begin{array}{l}\text { Anomalias cromossômicas, excluindo } \\
\text { mutações genéticas }\end{array}$ & $\begin{array}{l}\text { Q900, Q901, Q902, Q909, Q910, Q912, Q914, Q916, Q920, Q921, Q922, Q923, Q924, Q925, Q926, Q928, } \\
\text { Q929, Q927, Q930, Q931, D821, Q932, Q933, Q934, Q937, Q950, Q951, Q952, Q953, Q954, Q955, Q958, } \\
\text { Q959, Q960, Q961, Q962, Q963, Q964, Q968, Q969, Q970, Q971, Q972, Q980, Q981, Q984, Q985, Q986, } \\
\text { Q987, Q992, Q990, Q978, Q979, Q988, Q989, Q911, Q913, Q915, Q917, Q935, Q936, Q938, Q939, Q982, } \\
\text { Q983, Q991, Q998, Q999 }\end{array}$ \\
\hline $\begin{array}{l}\text { Condições com distúrbios do desenvol- } \\
\text { vimento intelectual como característica } \\
\text { clínica } \\
\text { relevante }\end{array}$ & F842, Q043, Q512, Q518, Q519, Q524, Q528, Q529, Q892, Q893, Q898, Q899 \\
\hline $\begin{array}{l}\text { Anomalias múltiplas ou síndromes do } \\
\text { desenvolvimento }\end{array}$ & 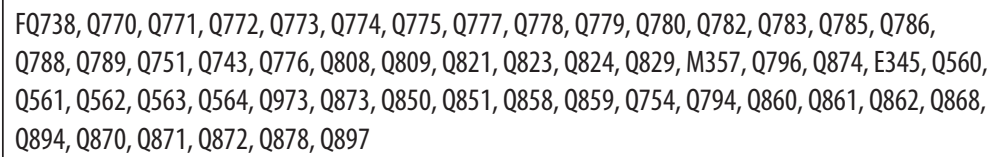 \\
\hline
\end{tabular}

Figura 2 - Classificação das anomalias congênitas de acordo com os capítulos da Classificação Estatística Internacional de Doenças e Problemas Relacionados à Saúde - Décima Primeira Revisão (CID-11)) 
continuação

\begin{tabular}{|c|c|}
\hline Classificação das anomalias & Códigos das anomalias \\
\hline $\begin{array}{l}\text { Anomalias estruturais do desenvolvimento } \\
\text { que afetam principalmente um sistema } \\
\text { corporal }\end{array}$ & 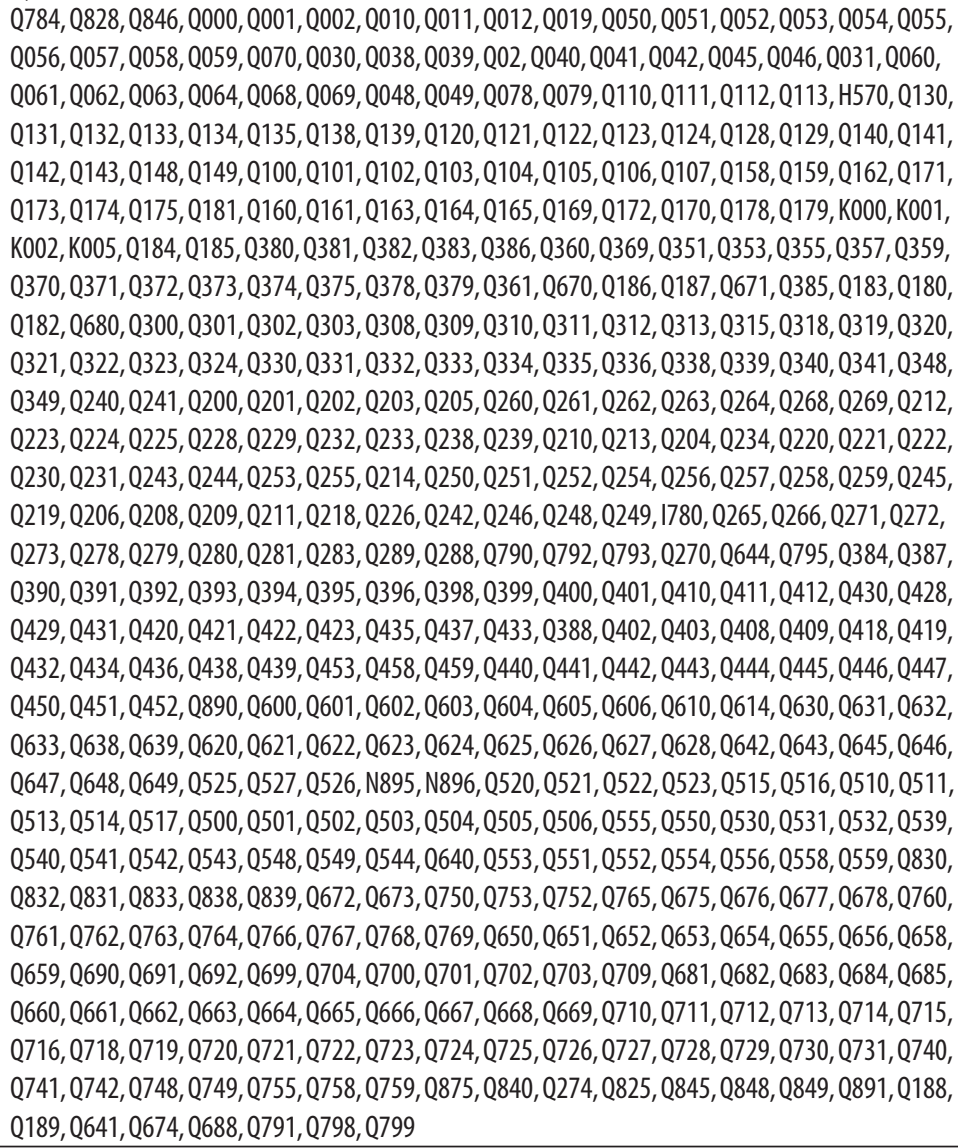 \\
\hline $\begin{array}{l}\text { Sintomas, sinais ou achados clínicos, não } \\
\text { classificados em outra parte }\end{array}$ & R011, R160, R161 \\
\hline
\end{tabular}

Figura 2 - Classificação das anomalias congênitas de acordo com os capítulos da Classificação Estatística
Internacional de Doenças e Problemas Relacionados à Saúde - Décima Primeira Revisão (CID-11)

\section{Discussão}

Este trabalho propõe uma lista dotada de 898 anomalias com códigos correspondentes na CID10, permitindo a instrumentalização do conceito de anomalias congênitas fornecido pela OMS. Comparada ao capítulo XVII da CID-10, ${ }^{6}$ a lista elaborada incluiu 279 novas condições. 0 Sinasc está habilitado para inclusão dos códigos constantes do capítulo XVII da CID-10, tão somente, o que limita a vigilância de anomalias congênitas no Brasil.

Uma lista compreensiva de anomalias congênitas codificadas na CID-10 torna-se importante para a vigilância. Sua codificação é amplamente utilizada pelos profissionais de saúde, para registro de casos nos sistemas de informações. Entretanto, o mapeamento dos códigos foi um desafio. Em muitos casos, estes autores não encontraram a correspondência exata entre os códigos das fontes de dados utilizadas e os da CID-10, o que pode conduzir a erros. Para minimizar essa limitação, apenas anomalias de correspondência exata com a CID-10 foram selecionadas e, posteriormente, realizada conferência manual dos códigos, no sentido de refinar sua acurácia.

A habilitação de novos códigos da CID-10 para inclusão de anomalias congênitas nos sistemas de informações, especialmente no Sinasc, será de extrema 


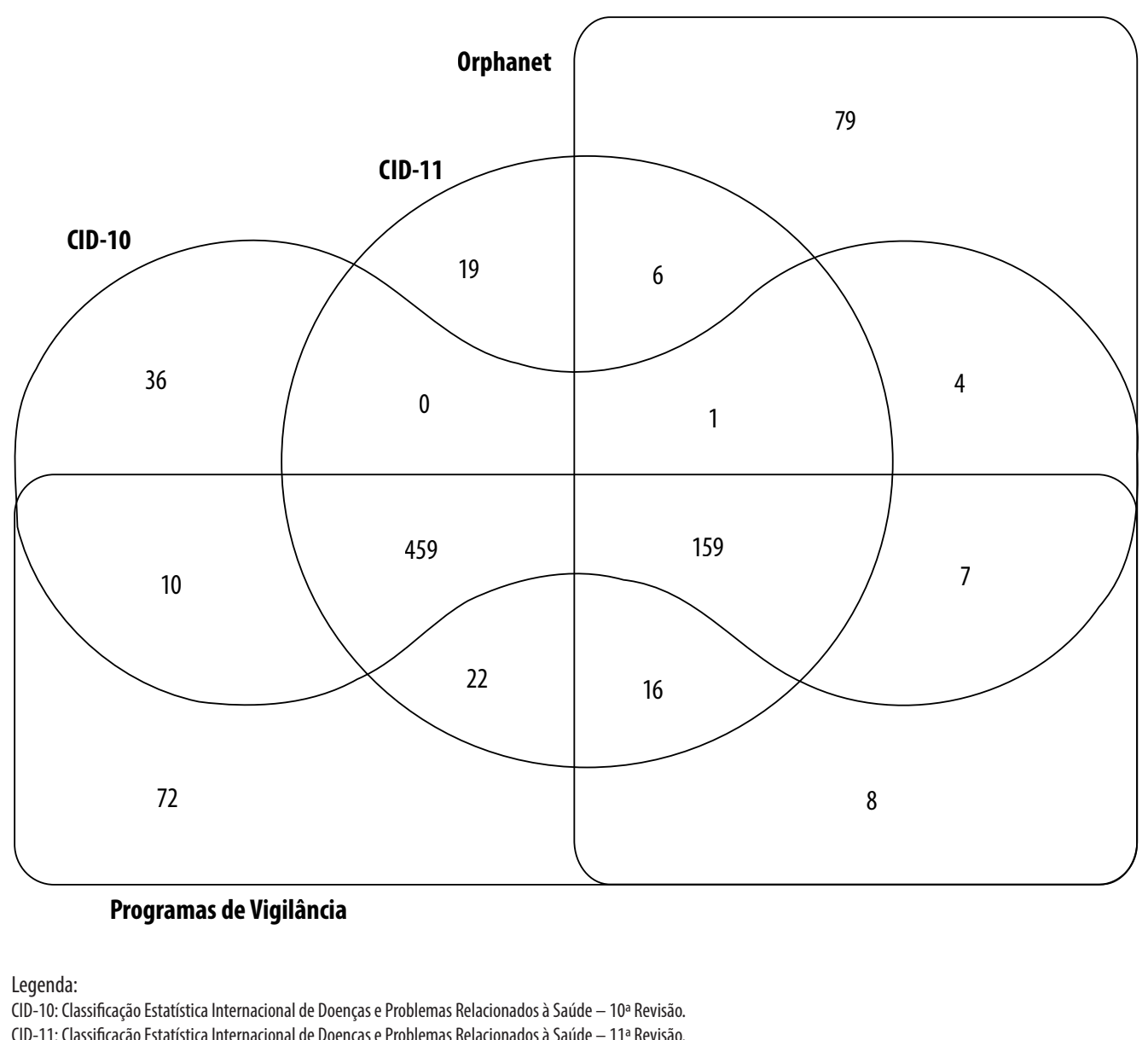

CID-11: Classificação Estatística Internacional de Doenças e Problemas Relacionados à Saúde - 11a Revisão.

\section{Figura 3 - Anomalias congênitas que se encontram na Classificação Estatística Internacional de Doenças e Problemas Relacionados à Saúde - Décima Revisão (CID-10), de acordo com as fontes de dados}

relevância para a vigilância em saúde. ${ }^{4} 0$ volume atual não dispões de codificação para algumas condições, e o capítulo referente às anomalias congênitas na CID-10 (XVII) se restringe, basicamente, às anomalias estruturais. Embora tenham-se somado novas condições ao capítulo 20 na CID-11, tal medida não foi suficiente para contemplar a totalidade de condições passíveis de definição como anomalias congênitas. Os dados extraídos e analisados corroboram a declaração conjunta das duas maiores redes internacionais para vigilância de anomalias congênitas, EUROCAT e ICBDSR. ${ }^{5}$

Nesse contexto, a lista expandida, aqui proposta, fornece um escopo ampliado de anomalias congênitas, útil para os fins da vigilância dessas condições, seus objetivos e prioridades. Conclui-se que a incorporação de novas fontes de dados aproxima esta lista da totalidade de anomalias congênitas passíveis de registro no Brasil.

\section{Contribuição dos autores}

Bremm JM, França GVA, Cardoso-dos-Santos AC, Magalhães VS, Alves RFS, Araujo VEM, Medeiros-deSouza AC, Oliveira WK, Macario EM, Schuler-Faccini L e Sanseverino MTV contribuíram com a concepção e delineamento do estudo, análise e interpretação dos dados. Bremm JM elaborou e fez a revisão crítica do manuscrito. Todos os autores revisaram criticamente 0 manuscrito, aprovaram sua versão final e declaram-se responsáveis por todos os aspectos do trabalho, incluindo a garantia de sua precisão e integridade. 


\section{Referências}

1. World Health Organization - WHO. Birth defects surveillance atlas of selected congenital anomalies [Internet]. Geneva: World Health Organization; 2014 [cited 2020 Aug 31]. 28 p. Available from: https://apps.who.int/iris/ handle/10665/127941

2. Harris BS, Bishop KC, Kemeny HR, Walker JS, Rhee E, Kuller JA. Risk factors for birth defects. Obstet Gynecol Surv [Internet]. 2017 Feb [cited 2020 Aug 31];72(2):123-35. Available from: https://doi.org/10.1097/ ogx.0000000000000405

3. Carmichael SL. Birth defects epidemiology. Eur J Med Genet [Internet]. $2014 \mathrm{Aug}$ [cited $2020 \mathrm{Aug}$ 31];57(8):355-8. Available from: https://doi.org/10.1016/j.ejmg.2014.03.002

4. March of Dimes. March of Dimes global report on birth defects [Internet]. Arlington: March of Dimes; 2020 [cited 2020 Mar 10]. Available from: https:/www. marchofdimes.org/mission/march-of-dimes-globalreport-on-birth-defects.aspx

5. EUROCAT, ICBDSR. Statement of EUROCAT and ICBDSR on ICD11 [Internet]. [S.1.]: EUR0CAT; 2009 [cited 2020 Jan 28]. Available from: https://eu-rd-platform. jrc.ec.europa.eu/sites/default/files/EUROCAT-JointStatement-ICD11-June-2009.pdf

6. Organização Mundial da Saúde - OMS. CID-10: Classificação estatística internacional de doenças. 10. ed. São Paulo: Edusp; 2007. (Com disquete, vol. 1).

7. Cardoso-dos-Santos A, Magalhães V, Medeiros-deSouza A, Bremm JM, Alves RFS, Araujo VEM, et al. Redes internacionais de colaboração para a vigilância das anomalias congênitas: uma revisão narrativa. Epidemiol Serv Saúde [Internet]. 2020 jul [citado 2020 ago 31];29(4):e2020093. Disponível em: https://doi. org/10.5123/s1679-49742020000400003
8. Orphanet. Orphanet: an online database of rare diseases and orphan drugs [Internet]. [S.1.]: Orphanet; 1997 [cited 2020 Jan 28]. Available from: https://www.orpha. net/consor/cgi-bin/index.php

9. Neu N, Duchon J, Zachariah P. TORCH infections. Clin Perinatol [Internet]. 2015 Mar [cited 2020 Aug 31];42(1):77103. Available from: https://doi.org/10.1016/j.clp.2014.11.001

10. Tucker FD, Morris JK, JRC Management Committee, Neville A, Garne E, Kinsner-0vaskainen A, et al. EUROCAT: an update on its functions and activities. J Community Genet [Internet]. 2018 0ct [cited 2020 Aug 31];9(4):407-10. Available from: https://doi.org/10.1007/s12687-018-0367-3

11. EUROCAT. EUROCAT Guide 1.4: Instruction for the registration of congenital anomalies [Internet]. Ispra: EUROCAT Central Registry; University of Ulster; 2013 [cited 2020 Jan 28]. Available from: https://eu-rd-platform.jrc.ec.europa.eu/sites/ default/files/Full_Guide_1_4_version_28_DEC2018.pdf

12. Poletta FA, Gili JA, Castilla EE. Latin American collaborative study of congenital malformations (ECLAMC): a model for health collaborative studies. Public Health Genomics [Internet]. 2014 [cited 2020 Aug 31];17(2):61-7. Available from: https://doi.org/10.1159/000356568

13. ECLAMC. Congenital malformations browser [Internet]. [S.l.]: ECLAMC; 2013 [citado 2020 jan 28]. Disponível em: http://en.atlaseclamc.org/

14. Ministerio de la Salud de Costa Rica. Protocolo de vigilancia de defectos congénitos en Costa Rica [Internet]. San José; Ministerio de la Salud de Costa Rica; 2018 [citado 2020 jan 28]. Disponível em: https://www. ministeriodesalud.go.cr/index.php/vigilancia-de-lasalud/normas-protocolos-y-guias/malformacionescongenitas/3785-protocolo-de-vigilancia-de-defectoscongenitos-marzo-2018/fileAbstract 


\section{Abstract}

objective: To propose a list of congenital anomalies having corresponding codes in the International Statistical Classification of Diseases and Related Health Problems, 10th Revision (ICD-10), with the aim of applying it in health surveillance. Methods: In December 2019, the following data sources were searched: ICD-10; ICD-11; anomalies monitored by three surveillance programs; and a database of rare diseases (Orphanet). Anomalies were retrieved from these data sources, processed to check for correspondence with ICD-10 and reviewed manually to compile the list. Results: 898 codes were identified, of which 619 (68.9\%) were contained in ICD-10 Chapter XVII. Of the 279 codes contained in other chapters, 19 were exclusive to the ICD-11 search, 72 to the surveillance programs, 79 to Orphanet and 36 to the search for terms in ICD-10. Conclusion: The codes contained in ICD-10 Chapter XVI do not capture the totality of congenital anomalies, indicating the need to adopt an expanded list.

Keywords: Congenital Abnormalities; Rare Diseases; International Classification of Diseases; Epidemiological Monitoring.

\section{Resumen}

objetivo: Proponer una lista de anomalías congénitas con códigos correspondientes en la décima revisión de la Clasificación Internacional de Enfermedades (CIE), con el objetivo de su aplicación en el ámbito de la vigilancia de la salud. Métodos: En diciembre de 2019, se buscaron las siguientes fuentes: CIE-10; CIE-11; anomalias monitoreadas por tres modelos de vigilancia; $y$ base de informaciones sobre enfermedades raras (Orphanet). Las anomalías se extrajeron de estas fuentes de datos, se procesó en base a la CIE-10 y se compiló con una revisión manual. Resultados: Se identificaron 898 códigos, de los cuales 619 (68,9\%) estaban en el Capítulo XVII de la CIE-10. De los 279 códigos en otros capítulos, 19 fueron exclusivos de la búsqueda en la CIE-11, 72 de los modelos de vigilancia, 79 de Orphanet y 36 de la búsqueda de términos en la CIE-10. Conclusión: Los códigos contenidos en el capítulo XVII de la CIE-10 no capturan la totalidad de las anomalías congénitas, lo que indica la necesidad de adoptar una lista ampliada.

Palabras clave: Anomalías Congénitas; Enfermedades Raras; Clasificación Internacional de Enfermedades; Monitoreo Epidemiológico.

Recebido em 14/04/2020

Aprovado em 14/07/2020 